\title{
Center frequency shift and reduction of feedback in directly modulated external cavity
} lasers

Schiellerup, G.; Pedersen, Rune Johan Skullerud; Olesen, H.; Tromborg, B.

Published in:

I E E E Photonics Technology Letters

Link to article, DOI:

10.1109/68.43351

Publication date:

1989

Document Version

Publisher's PDF, also known as Version of record

Link back to DTU Orbit

Citation (APA):

Schiellerup, G., Pedersen, R. J. S., Olesen, H., \& Tromborg, B. (1989). Center frequency shift and reduction of feedback in directly modulated external cavity lasers. I E E E Photonics Technology Letters, 1(10), 288-290. https://doi.org/10.1109/68.43351

\section{General rights}

Copyright and moral rights for the publications made accessible in the public portal are retained by the authors and/or other copyright owners and it is a condition of accessing publications that users recognise and abide by the legal requirements associated with these rights.

- Users may download and print one copy of any publication from the public portal for the purpose of private study or research.

- You may not further distribute the material or use it for any profit-making activity or commercial gain

- You may freely distribute the URL identifying the publication in the public portal 


\title{
Center Frequency Shift and Reduction of Feedback in Directly Modulated External Cavity Lasers
}

\author{
G. SCHIELLERUP, R. J. S. PEDERSEN, H. OLESEN, AND B. TROMBORG, MEMBER, IEEE
}

\begin{abstract}
We show experimentally and theoretically that a center frequency shift occurs when an external cavity laser is directly modulated. The shift may be observed even when the frequency deviation is small compared to the roundtrip frequency of the external cavity and can qualitatively be explained by a reduction in the effective feedback level due to modulation. The frequency shift has been measured as a function of modulation frequency and current, and frequency shifts up to 350 MHz were observed.
\end{abstract}

\section{INTRODUCTION}

I IS well known that semiconductor lasers with external feedback can meet the requirement for low linewidth in coherent optical communication systems. Unfortunately, external feedback reduces the overall level of FM response and introduces additional resonance peaks [1], [2]. In this letter, we show that direct frequency modulation of an external cavity laser also results in a reduced effective feedback level, leading to a center frequency shift compared to the unmodulated laser. To our knowledge, this unwanted effect has not previously been reported. The phenomenon can be understood in simple terms. An AR-coated Fabry-Perot laser with optical feedback will usually oscillate in the external cavity mode with lowest carrier density. The reduction in carrier density leads to an increase in refractive index and hence a reduction in oscillation frequency. By modulating the laser, the level of coherent feedback is reduced which results in a smaller reduction of the oscillation frequency. Thus, modulation leads to a positive frequency pulling. We have modeled the effect by use of an optical transmission line theory described in [3].

\section{EXPERIMENTAL RESULTS}

The spectrum of a modulated external cavity laser was observed using a scanning Fabry-Perot interferometer setup (see Fig. 1). The setup includes two optical isolators with a total isolation of approximately $50 \mathrm{~dB}$. The external cavity configuration consists of a $1.3 \mu \mathrm{m}$ Fabry-Perot laser with one facet cleaved and the other facet AR-coated to a reflectivity of approximately 1 percent and a $6 \mathrm{~cm}$ long external cavity with a 600 line/mm grating. The laser has a threshold of $44 \mathrm{~mA}$ at a feedback power ratio of approximately 20 percent. In the experiment, the laser was biased at $60 \mathrm{~mA}$. The $\mathrm{FM}$ response

Manuscript received June 22, 1989; revised July 13, 1989.

G. Schiellerup and R. J. S. Pedersen are with Center for Broadband Telecommunications, Electromagnetics Institute, Technical University of Denmark, DK-2800 Lyngby, Denmark.

H. Olesen and B. Tromborg are with the TFL Telecommunications Research Laboratory, DK-2970 Horsholm, Denmark.

IEEE Log Number 8930898. can be determined from the relative height of the modulation sidebands [4]. It was observed that the carrier frequency shifted towards higher frequencies as the modulation was turned on. The shift is denoted by $\delta f$ in the following.

The frequency shift shows a strongly resonant character when measured as a function of modulation frequency, and a nonlinear behavior when measured as a function of modulation current. Fig. 2 shows the frequency shift as a function of modulation frequency in the range from dc to $1 \mathrm{GHz}$. The amplitude $I_{m}$ of the modulation current was held constant at 2 $\mathrm{mA}$. Fig. 3 shows the frequency shift as a function of modulation current at a modulation frequency of $280 \mathrm{MHz}$. It should be noted that the amount of frequency shift depends critically on the adjustment of the external cavity. Figs. 2 and 3 are therefore not directly comparable. Both larger and smaller values could be obtained, but in all cases the resonant character and nonlinear behavior were observed. The setup was carefully checked for parasitic optical and electrical resonators.

\section{THEORY}

The observed behavior can qualitatively be explained by the optical transmission line model presented in [3]. The rate equation for the photon number $I(t)$ and the phase $\varphi(t)$ is given by (cf. [3, eq. (22)])

$$
\begin{aligned}
\frac{d I}{d t}+j 2 I(t) \frac{d \varphi}{d t}-(1+j \alpha) & G_{N} \Delta N(t) I(t) \\
= & 2 f_{D}\left\{\sigma r_{L} A^{-}(t)\left[A^{+}(t)\right]^{*}-I(t)\right\}
\end{aligned}
$$

where $A^{+}(t)$ is the envelope function of the right-going field at a reference plane just inside the AR-coated facet. The leftgoing field at the reference plane is $A^{-}(t)=\rho(t) \otimes A^{+}(t)$ where $\rho(t)$ is the impulse response of the external cavity and $\otimes$ denotes convolution. $\Delta N$ is the change in carrier density compared to the unmodulated laser, $\alpha$ is the linewidth enhancement factor, $G_{N}$ is a gain constant, $f_{D}$ is the roundtrip frequency of the diode cavity, $\sigma$ is a proportionality constant, and $r_{L}$ is the effective reflectivity of the Fabry-Perot laser section seen towards the left from the reference plane. Spontaneous emission and phase noise are neglected in the following.

In the case with sinusoidal modulation, we have [4]

$$
A^{+}(t)=A \sqrt{1+m \sin \left(\omega_{m} t\right)} e^{j\left[\delta \omega t+\beta \cos \left(\omega_{m} t+\theta\right)\right]}
$$

where $A$ is a constant, $m$ is the intensity modulation index, $\omega_{m}$

$1041-1135 / 89 / 1000-0288 \$ 01.00$ (C) 1989 IEEE 


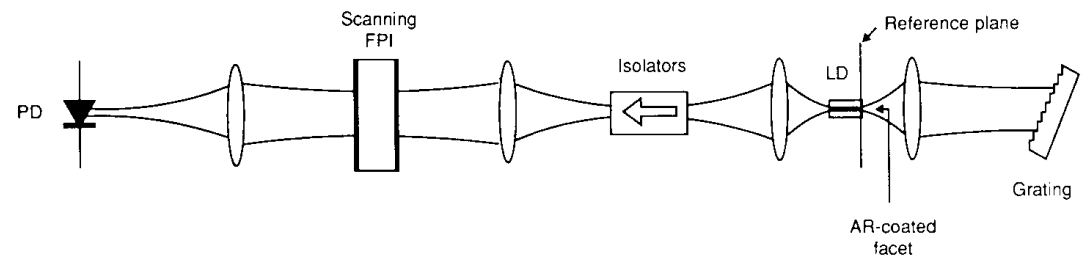

Fig. 1. Measurement setup for measuring the FM response. Fabry-Perot parameters: $F S R=300 \mathrm{MHz}, F \approx 100$.

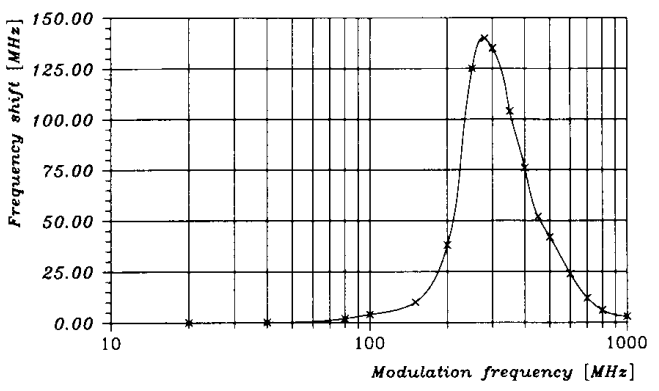

Fig. 2. Measured frequency shift $\delta f$ versus modulation frequency $f_{m}$ at $I_{m}$ $=2 \mathrm{~mA}$.

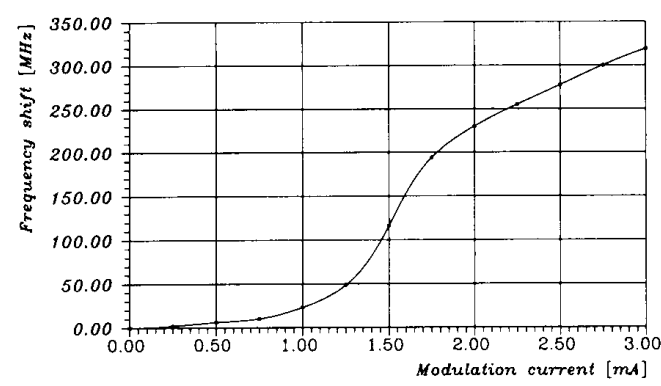

Fig. 3. Measured frequency shift $\delta f$ versus modulation current $I_{m}$ at the modulation frequency $f_{m}=280 \mathrm{MHz}$.

is the angular modulation frequency $\left(=2 \pi f_{m}\right), \beta$ is the FM index, $\theta$ is the phase delay between IM and FM, and $\delta \omega=$ $2 \pi \delta f$.

In order to determine the average frequency shift due to modulation, we insert (2) in (1) and take the time average over one modulation period. After some mathematics we obtain

$$
\begin{aligned}
\delta \omega=-f_{D}\left[\alpha \ln \left|\frac{r_{R}^{\prime}\left(\omega_{s}+\delta \omega\right)}{r_{R}\left(\omega_{s}\right)}\right|-\operatorname{Arg}\{\right. & \left.r_{R}^{\prime}\left(\omega_{s}+\delta \omega\right)\right\} \\
& \left.+\operatorname{Arg}\left\{r_{R}\left(\omega_{s}\right)\right\}\right]
\end{aligned}
$$

where $\omega_{s}$ is the oscillation frequency without modulation and $r_{R}$ is the effective reflectivity of the external cavity seen from the reference plane without modulation. The relation between $r_{R}(\omega)$ and the previously introduced impulse response $\rho(t)$ is given by

$$
\rho(t)=\sum_{n=0}^{\infty} \rho_{n} e^{-j n \omega_{s} \tau} \delta(t-n \tau)=\frac{1}{2 \pi} \int_{-\infty}^{\infty} r_{R}(\omega) e^{j\left(\omega-\omega_{S}\right) t} d \omega
$$

where $\rho_{n}$ is the strength of the $n$th reflection.

The corresponding effective reflectivity with modulation is defined by

$$
r_{R}^{\prime}\left(\omega_{s}+\delta \omega\right)=\left|\frac{\rho(t) \otimes A^{+}(t)}{A^{+}(t)}\right|
$$

where $\langle\cdots\rangle$ denotes time average over one modulation period. In the case of pure sinusoidal frequency modulation, (5) can be written as

$$
r_{R}^{\prime}\left(\omega_{s}+\delta \omega\right)=\sum_{n=0}^{\infty} \rho_{n} J_{0}\left(2 \pi n \Delta f \tau \frac{\sin \left(\pi n f_{m} \tau\right)}{\pi n f_{m} \tau}\right) e^{-j n\left(\omega_{S}+\delta \omega\right) \tau}
$$

where $J_{0}(x)$ is the Bessel function of order zero, and $\Delta f=$ $\beta f_{m}$ is the peak frequency deviation. In practice, only the first few terms in the summation are significant.

A comparison of (6) to (4) shows that in the case of modulation the $n$th term in the summation is weighted by a factor less than one. The feedback level will thereby be reduced compared to the unmodulated case leading to a frequency shift towards the laser frequency without external feedback. From (6) it can also be seen that the frequency shift will occur even for short cavity lasers such as monolithically integrated devices, if these are used in high bit rate FSK systems. In this case, the decrease in $\tau$ can be compensated by the larger frequency deviation which is required in order to have a sufficiently large FM index.

\section{Results ANd Discussion}

In order to compare the measured data in Fig. 2 with theory, it would be desirable to have a model for the FM response of the external cavity laser, which takes into account the variation of $r_{R}^{\prime}$. However, this model has not yet been developed, and we have instead evaluated (3) as a function of modulation frequency $f_{m}$ and frequency deviation $\Delta f$. We have used the following parameters: $m=0.1, \theta=0, r_{R}\left(\omega_{s}\right)=0.62, \alpha=$ 6.6. Fig. 4 shows the result of this calculation together with measured frequency shift versus modulation frequency, from Fig. 2, projected onto the surface. From this projection, we can read off the corresponding frequency deviation versus modulation frequency. The resulting FM response is shown in Fig. 5, and the shape compares well with observations in the laboratory. The coupling between the frequency shift and the FM response can be explained physically by their common dependency on $r_{R}^{\prime}(\omega)$. Because $\left|r_{R}^{\prime}(\omega)\right|$ is decreasing with increasing $\Delta f$, and $\Delta f$ is increasing with decreasing $\left|r_{R}^{\prime}(\omega)\right|$, the system forms a positive feedback loop that causes the resonance seen in Fig. 2. An attempt to verify the theory by measuring the frequency shift as a function of $f_{m}$ with constant 


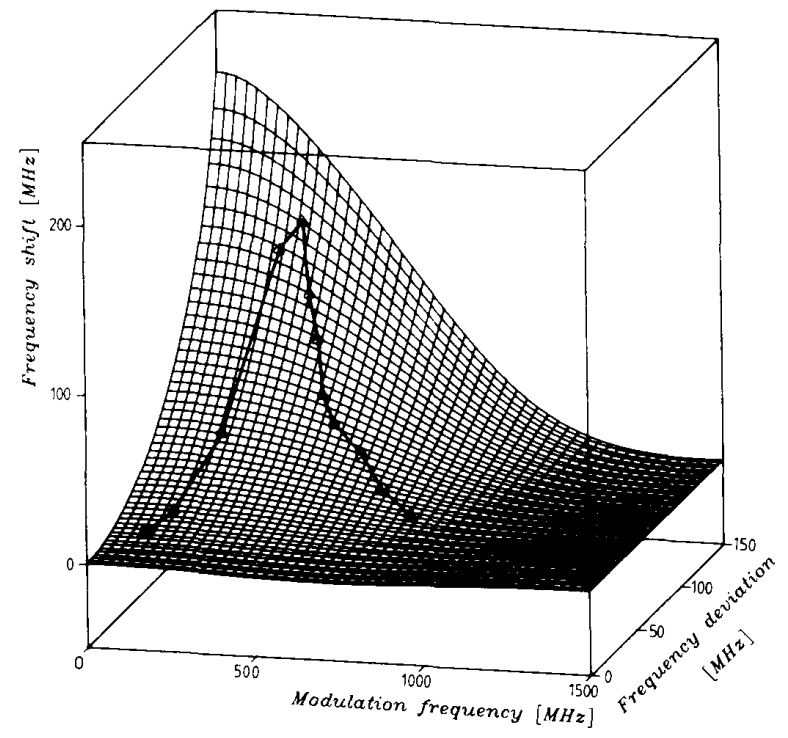

Fig. 4. Calculated frequency shift $\delta f$ versus modulation frequency $f_{m}$ and frequency deviation $\Delta f$. The solid line indicates the measured curve from Fig. 2.

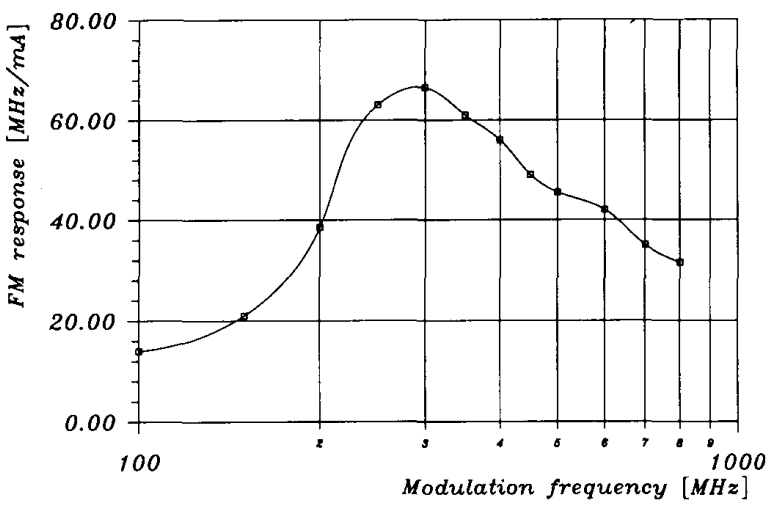

Fig. 5. FM response $\Delta f / I_{m}$ obtained from Fig. 4 .
$\Delta f$, thus testing the theory without the coupling to the FM response, failed due to modulation-induced mode hopping.

The positive feedback mechanism complicates the use of the external cavity laser as transmitter in coherent systems, because FM response becomes very sensitive to small changes in the external cavity laser parameters. Another problem is the frequency shift itself, which will introduce a slowly time varying carrier frequency when the laser is modulated with a random bit stream. One might therefore expect a pattern dependent bit error rate.

\section{CONCLUSION}

Our measurements show that a center frequency shift occurs when an external cavity laser is frequency modulated. This might lead to pattern effects if the laser is used as transmitter in a digital coherent optical communication system.

A model based on a transmission line description of the laser has been developed. Theoretical predictions of the frequency shift of a frequency modulated external cavity laser agree qualitatively with measured results. The model shows that monolithically integrated external cavity lasers will show similar behavior when used in high bit rate FSK-systems.

\section{ACKNOWLEDGMENT}

The authors are grateful to G. Jacobsen and E. B $\varnothing$ dtker from TFL Telecommunications Research Laboratory for their encouragement during this work.

\section{REFERENCES}

11] S. Saito, O. Nilsson, and Y Yamamoto, "Oscillation center frequency tuning, quantum FM noise, and direct frequency modulation characteristics in external grating loaded semiconductor lasers," IEEE $J$ Quantum Electron., vol. QE-18, pp. 961-970, June 1982.

[2] A. J. Lucero, R. W. Tkach, and R. M. Derosier, "Distortion of the frequency modulation spectra of semiconductor lasers by weak optical feedback," Electron. Lett., vol. 24, pp. 337-339, Mar. 1988.

[3] B. Tromborg, H. Olesen, X. Pan, and S. Saito, "Transmission line description of optical feedback and injection locking for Fabry-Perot and DFB lasers," IEEE J. Quantum Electron., vol. QE-23, pp. 1875-1889, Nov. 1987.

[4] H. Olesen and G. Jacobsen, "A theoretical and experimental analysis of modulated laser fields and power spectra," IEEE J. Quantum Electron., vol. QE-18, pp. 2069-2080, Dec. 1982. 